\title{
Dimensional analysis of droplet size and ligament length during high-speed rotary bell atomization
}

\author{
Lutz Gödeke $\mathbb{1}$, Walter Oswald, Norbert Willenbacher, Peter Ehrhard
}

(C) The Author(s) 2020

\begin{abstract}
Modern spray-coating processes are based on high-volume, low-pressure, airless atomization or high-speed rotary bell atomization, often assisted by electrostatic charging to increase the transfer efficiency. The process from the liquid film flow beneath the bell, through ligament formation and consecutive disintegration to droplet deposition, has been constantly explored during the evolution of automotive spray coating. This work proposes a set of dimensionless groups that fully describe the process from film flow to ligament disintegration, including shear and elongational flow effects during atomization of particle laden, shear thinning, viscoelastic fluids.
\end{abstract}

Keywords Dimensional analysis, High-speed rotary bell atomization, Elongational flow, Ligament disintegration, Droplet size, Acrylic thickener, Plate-like particles

\section{Introduction}

High-speed rotary bell atomization is the state-of-theart technology for paint application in the automotive industry. In recent years, research on this topic has been published in experimental, theoretical, and numerical form, dealing with both the formation of droplets and the measurement methods used. Funda-

L. Gödeke $(\bowtie)$, P. Ehrhard

Fluid Mechanics, Biochemical and Chemical Engineering, TU Dortmund, Emil-Figge-Strasse 68, 44227 Dortmund, Germany

e-mail: lutz.goedeke@tu-dortmund.de

W. Oswald, N. Willenbacher

Applied Mechanics, Institute of Mechanical Process Engineering and Mechanics, KIT, Gotthard-Franz-Straße 3, 76131 Karlsruhe, Germany mental work has been done by Hinze and Milborn. ${ }^{1}$ Their publication deals with the atomization of an idealized film flow and ligament disintegration of Newtonian liquids for nonserrated bells. The process is well described starting with an analytical solution for the film flow and leading to a thorough analysis of the ligament formation with experimental data validation. Domnick et al. ${ }^{2}$ used measurement data as a basis for numerical models to predict the spraying process. Mescher ${ }^{3}$ and Kamplade et al. ${ }^{4}$ carried out experiments on ligament disintegration at transverse flow and subsequent numerical investigations to optimize the gas flow with respect to the resulting droplet size distribution. Kalmbach et al. $^{5}$ used a comparable theoretical approach and validated the asymptotic solutions of linear stability analysis with experiments. A detailed analytical investigation of the resulting drop size from an emerging jet of a rivulet inside a bore of a spinning cup was carried out by Kuhnhenn et al. ${ }^{6}$ The obtained results for water droplets, under consideration of the appearing strain rate $\dot{\varepsilon}$, provide a good prediction for the experimental data by using a function of the capillary breakup time after Rayleigh ${ }^{7}$ and the strain rate $\dot{\varepsilon}$. Furthermore, Tratnig et al. ${ }^{8}$ investigated the pressure-swirl atomization of various particle-laden opaque Carreau-Yasuda liquids by means of phase Doppler anemometry (PDA). The results were then summarized in a product of power laws.

The entirety of the aforementioned studies has contributed a lot to a better understanding of the process. However, the handling of viscoelastic particleladen fluids has not yet been investigated in detail with underlying experimental data. The model liquids used in this work exhibit complex rheological properties, consisting of different aqueous acrylic thickener solutions and glass particles. Their flow behavior was characterized in detail by Oswald and Willenbacher ${ }^{9}$, and atomization of these fluids was investigated by 
Oswald et al. ${ }^{10}$ During high-speed rotary bell atomization, the model liquids are conveyed into the interior of a rotating bell and form ligaments at the bell edge, which then disintegrate into fine droplets with characteristic size distributions. These distributions depend on the respective fluid properties such as density, surface tension, shear and extensional rheological characteristics and on process parameters such as rotational speed of the bell and volumetric flow of the liquid. During typical coating processes, these liquid droplets are transported toward the substrate with the aid of the so-called shaping gas flow, where they are deposited on the substrate and form a thin film, due to coalescence and surface wetting. The determination of the relevant process parameters and material properties is necessary for a detailed understanding of the entire process.

Conventionally measured liquid properties (e.g., CaBER and low-shear viscosity measurement) do not deliver the full data needed for the description of ligament buildup and disintegration into droplets. The use of high-speed imaging to detect ligament length and a simultaneous measurement of droplet sizes using defined model liquids offers the possibility to analyze the interlocked effects, under consideration of liquidparticle interactions and the effect of viscoelasticity during operating conditions. Electrostatic charging, which is primarily used to increase transfer efficiency, was not a part of the experimental investigation, as the setup of high-speed camera and PDA did not allow for a sufficient safety clearance in order to prevent electric arcing. Additionally, there have been no indications in preemptive measurements that electrostatic charging influences the drop size distribution in proximity of the bell edge.

\section{General process description and dimensional analysis}

The overall process can be divided into three major portions: (i) the film flow beneath the bell, (ii) the formation of ligaments, leading to their disintegration and the resulting formation of droplets, and finally (iii) the flight phase with subsequent droplet impact on the solid or liquid surfaces. The used rotary bell atomizer injects the liquid to the inner side of the spinning bell through a centered channel in the rotating shaft. A thin film of liquid is formed due to strong centrifugal forces, which then emerges from the bell edge, either by directly forming droplets, as well as ligaments or different forms of lamellae that consecutively disintegrate into droplets. The desirable type of droplet formation for most technical processes is the ligament disintegration, as it leads to defined droplet size distributions with narrow span. For smooth-edged bells, the ligament properties (ligament spacing, initial diameter) are a result of flow instabilities that induce specific flow patterns depending on liquid properties and process parameters. This naturally leads to a partially chaotic behavior, due to small disturbances in the film. A defined variation of this ligament buildup is realized by the use of serrated bells. Small triangular channels are cut into the edge of the bell, which force the liquid film to split into a defined number of rivulets, which consecutively discharge as ligaments. In contrast to smooth-edged bells, these serrations will maintain a constant number of emerging ligaments during the variation of process parameters within the limits of the ligament disintegration mode. The discharge velocity and ligament diameter will change according to volumetric flow rate, bell dimension, and rotational speed.

The dimensional analysis offers the possibility of significantly reducing the number of measurements in order to obtain desired correlations by organizing the influence parameters of a process in dimensionless groups. The correlation based on the dimensionless groups, thus, becomes generally valid, as long as the underlying physics is reflected correctly. The procedure is common for modeling problems and can be achieved by the use of Buckingham's $\Pi$ theorem. ${ }^{11}$

The droplet formation during bell atomization is a result of disintegrating paint ligaments. Therefore, it is necessary to identify the physical parameters that act at this location correctly for the dimensional analysis. The initial diameter of the ligaments $d_{L i g, 0}$ varies weakly with relation to varying process conditions and is approximately of the order of the serration cross-sectional diameter $d_{S e r}$. The process and material parameters considered in the dimensional analysis are defined at the bell edge and, therefore, primarily influence the length of the ligament emerging. In ambient air, the ligament breaks down into droplets, the average size of which is dependent on a critical ligament diameter that can depend on the initial ligament diameter $d_{L i g, 0}$ and on the ligament length $l_{\text {Lig }}$. The ligament length and the droplet diameter both can be used as target values for the dimensional analysis.

The major geometrical dimensions are given by $d_{p}$ as the droplet diameter, $d_{S e r}$ as the characteristic measure of the serration (e.g., hydraulic diameter), $l_{\text {Lig }}$ as ligament length, and $R$ as bell radius. The process parameters are the bell speed $\omega$ and the liquid volumetric flow rate $\dot{V}$, which is implemented as an axial ligament flow velocity $c_{a x}$ using equation (1). Under the assumption of an ideal liquid distribution between the entirety of $N$ different equilateral triangular serrations with a flat rivulet and an initially undisturbed rotationally symmetric ligament, we obtain

$$
c_{a x}=\frac{4 \dot{V}}{\sqrt{3} \cdot d_{S e r}^{2} \cdot N} .
$$

This simplified solution yields a good estimate of the initial average velocity inside the ligament, yet does not take into account disturbances of the free surface inside the serration rivulet or local velocity distributions, as it is based on an integral balance. 
However, the axial velocity for the disturbed surface is directly proportional to $c_{a x}$; therefore, the dependency on $\dot{V}$ is valid for the product of power laws. For constant ambient conditions and moderate bell speed, both gas viscosity $\eta_{g}$ and gas density $\rho_{g}$ are treated as constant. Surface tension $\sigma$ and liquid density $\rho_{l}$ are assumed to be constant. The rheological behavior of the liquid phase is characterized by a shear and an elongational relaxation time $\left(\lambda_{s}, \lambda_{e}\right)$ and by shear and elongational viscosities $\left(\eta_{s}, \eta_{e}\right)$ at a constant given shear $\left(\dot{\gamma}=3 \times 10^{5} s^{-1}\right)$ and strain rate $(\dot{\varepsilon})$. Both relaxation times of viscoelastic liquids can be obtained experimentally as described by Macosko. ${ }^{12}$ The amount of glass flakes of density $\rho_{p}$ defines the solid content of the liquid phase $\phi$, which serves as an additional dimensionless group. Applying Buckingham's $\Pi$ theorem for the given system, a set of dimensionless groups can be derived that yields the general form

$$
\Pi_{\sum}=f\left(d_{p}, d_{S e r}, l_{L i g}, R, \omega, c_{a x}, \sigma, \lambda_{e}, \lambda_{s}, \eta_{e}, \eta_{s}, \eta_{g}, \rho_{l}, \rho_{g}, \rho_{g}, \phi\right) .
$$

However, the approach of using a defined elongational viscosity for the definition of dimensionless groups is problematic. Typically $\eta_{e}$ is obtained from the CaBER experiment $\left(\dot{\varepsilon}_{\max }=100 s^{-1}\right)$ and, therefore, calculated using the surface tension $\sigma$ and the rate of deformation as shown in Stelter et al. ${ }^{13}$ Based upon the rate of deformation, these values may vary by several orders of magnitude. Recent work of Kuhnhenn et al. ${ }^{6}$ provides a solution for a defined rivulet flow emerging from a cylindrical bore. According to the mathematical definition of the strain rate, we expect strain rates ranging from $\dot{\varepsilon}=1000-7000 s^{-1}$ for the used rotary atomizer. Therefore, the CaBER device does not cover the strain rates during high-speed bell atomization. For the given case, we do not have a precise way of calculating the strain rate $\dot{\varepsilon}$, as the exact position of the free surface and, therefore, the exact flow field is unknown. Accordingly, we neglect the elongational viscosity $\eta_{e}$ and obtain twelve dimensionless groups to characterize the general breakup process at constant ambient conditions $\left(T=\right.$ const.,$\varphi=$ const.,$p_{0}=$ const.$)$, i.e.,

$$
\begin{aligned}
\Pi_{\sum}= & g\left(\Pi_{1}, \dot{s}, \Pi_{12}\right) \\
\Pi_{\sum}= & g\left(\frac{l_{L i g}}{d_{S e r}}, \frac{d_{p}}{d_{S e r}}, \frac{\rho_{g}}{\rho_{l}}, \frac{\eta_{g}}{\eta_{s}}, \frac{d_{S e r}}{R}, \frac{\lambda_{e}}{\lambda_{s}}, \frac{c_{a x}}{\omega \cdot R}, \frac{c_{a x} \cdot \rho_{l} \cdot d_{S e r}}{\eta_{s}}, \frac{c_{a x}^{2} \cdot \rho_{l} \cdot d_{S e r}}{\sigma},\right. \\
& \left.\lambda_{e} \cdot\left(\frac{\sigma}{\rho_{l} \cdot d_{S e r}^{3}}\right)^{0.5}, \frac{\rho_{p}}{\rho_{l}}, \phi\right) .
\end{aligned}
$$

Here $\Pi_{1}-\Pi_{6}$ and $\Pi_{11}$ are ratios of parameters of equal dimensions, $\Pi_{7}$ is the ratio of axial liquid velocity inside the ligament and tangential velocity at the bell edge and, therefore, defining the stretching characteristics of the ligament flow. $\Pi_{8}$ is a liquidrelated Reynolds number inside the ligament at its origin $\left(\operatorname{Re}_{a x, l}\right)$, formed with the constant shear viscosity at the bell edge and axial ligament flow velocity. $\Pi_{9}$ is a liquidrelated Weber number inside the ligament $\left(\mathrm{We}_{a x, l}\right)$, formed with the axial ligament flow velocity and $\Pi_{10}$ is a Deborah number formed with a characteristic breakup time. Both the Reynolds and the Weber number can be formed as corresponding gas-related dimensionless groups. The Deborah number can be formed with different characteristic timescales relative to a specific relaxation time. For the given case, the capillary breakup time $t_{\text {cap }}$ is chosen as the liquid-specific timescale. Hence, we obtain

$$
\begin{aligned}
t_{\text {cap }} & =\left(\frac{\rho_{l} \cdot d_{\text {Ser }}^{3}}{\sigma}\right)^{0.5}, \\
\Pi_{10} & =\frac{\lambda_{e}}{t_{c a p}}=D e_{\text {cap }} .
\end{aligned}
$$

Dynamic and viscous timescales can be obtained through dimensional analysis by choosing the corresponding basis. The ideal timescale for the ligament breakup is the actual breakup time $t_{L i g}=l_{L i g} \cdot c_{a x}{ }^{-1}$, which is a function of the axial velocity $c_{a x}$ and one of the two target parameters $l_{\text {Lig }}$ and, therefore, not available as a variable. For the majority of technically used paint liquids $\Pi_{6} \rightarrow 0$, the shear relaxation time $\left(\lambda_{s} \approx 10^{2}-2 \times 10^{4} s\right)$ is larger by several orders of magnitude compared to the elongational relaxation time $\left(\lambda_{e} \approx 10^{-2}-5 \times 10^{-1} s\right)$ as described in Oswald and Willenbacher. ${ }^{9}$ Additionally, for these liquids $t_{\text {Lig }} \ll \lambda_{s}$ holds, therefore, we neglect the influence of shear relaxation on ligament breakup.

To account for the process relevant shear viscosity, we estimate the shear rate $\dot{\gamma}$ at the bell edge by approximating a simplified flow field for the local velocity distribution normal to the bell surface inside the film assuming rotational symmetry. The implementation of a rheology model is fulfilled by using an iteration loop for the film thickness and flow velocity until the obtained solutions converge. The local shear gradient is averaged over the film thickness to obtain the mean shear gradient. A similar method is described by Weckerle. ${ }^{14}$ However, this approach neglects surface effects, film disturbances, and liquid inhomogeneities and has to be further specified by solving the actual three-dimensional flow for the given rheological model to obtain a more accurate result. The estimation shows that the shear gradients for commonly used process and liquid parameters are of the order $10^{4}-5 \times 10^{5} s^{-1}$. Unconditionally, the film thickness and local velocity calculated by Hinze and Milborn ${ }^{1}$ can be used to infer the average shear gradient for Newtonian liquids. The automotive coatings investi- 
gated are shear thinning, but approach a high-shear viscosity $\eta_{S, \infty}\left(\dot{\gamma}=3 \times 10^{5} s^{-1}\right)$ of about 15-30 MPas.

The dimensionless groups can be used in product of power laws to find correlations for the ligament length and the droplet size, both depending on process parameters, as well as material properties by displaying $\Pi_{1}$ and $\Pi_{2}$ as functions of the remaining groups, namely

$$
\begin{aligned}
& \Pi_{1}=\frac{l_{\text {Lig }}}{d_{\text {Ser }}}=\prod_{i=3}^{10} \Pi_{i}^{a_{i}}, \\
& \Pi_{2}=\frac{d_{p}}{d_{\text {Ser }}}=\prod_{j=3}^{10} \Pi_{j}^{a_{j}} .
\end{aligned}
$$

\section{Experimental results and discussion}

The used liquids $\left(S_{1}-S_{5}\right)$ are model systems based on aqueous solutions of Sterocoll HT and Sterocoll XT (BASF SE, Ludwigshafen, Germany). Shear properties are set using different concentrations of Sterocoll HT (SHT) and elongational properties are controlled by the addition of Sterocoll XT (SXT). Sample $S_{2}$ is mixed with different amounts of glass flakes $\left(d_{v, 50}=25.0-35.0 \mu \mathrm{m}, \rho_{p}=2600 \mathrm{~kg} / \mathrm{m}^{3}\right.$ Luxan CFX C001, Eckart GmbH, Hartenstein, Germany) to obtain liquids suspensions of different solid volume fraction $\phi$ $\left(S_{2} G_{1}\right.$ and $\left.S_{2} G_{2}\right)$. All liquid properties are shown in Table 1.

The atomizer is an EcoBell2 (Dürr AG, Stuttgart, Germany) with a serrated bell $(R=65 \mathrm{~mm}$, $\left.d_{S e r}=0.12 \mathrm{~mm}\right)$. Measurements are taken at constant ambient conditions $\left(T=25^{\circ} \mathrm{C}, \rho_{g}=1.293 \mathrm{~kg} / \mathrm{m}^{3}\right.$, $\varphi_{\text {air }}=65 \%$ ) in a clean room with a vertical gas velocity of $0.3 \mathrm{~m} / \mathrm{s}$. Liquid volume flow rate and bell speed are varied in the ranges $\dot{V}_{l}=200-350 \mathrm{~mL} / \mathrm{min}$ and $n=10$ $50 \mathrm{krpm}$. The shaping gas flow rate is held constant at $340 \mathrm{NL} / \mathrm{min}$. Previous work has shown no significant influence of the shaping gas flow rate on droplet size and ligament length. The ligament length is determined by image capturing at a frame rate of $25.000 \mathrm{fps}$ using the high-speed camera Fastcam SA-Z (Photron Tokyo, Japan) as described by Oswald et al. ${ }^{10}$ Droplet size is measured using SpraySpy (AOM-Systems GmbH, Heppenheim, Germany) along an axis from the center of the cone to the outer region. Subsequently, droplet sizes are averaged over the corresponding cross-sectional area of the spray for the arithmetic mean droplet diameter $D_{N, 10}$ of the number distribution as described by Tratnig et al. ${ }^{8}$ The addition of glass flakes to the transparent Sterocoll mixtures leads to a slight decrease of the local data rates. This could be compensated by a slight change of the device sensibility. However, this would lead to a change in the overall measurement characteristics. As the amount of validated detections for each segment of the measurement axis is sufficient to satisfy statistical certainty according to Adams et al., ${ }^{15}$ the device settings remain unchanged, though.

In Fig. 1 the scaled data sets obtained from multiple measurements are plotted against correlations (9) for $\Pi_{1, \text { total }}$ and (10) for $\Pi_{2, \text { total }}$. For each correlation, the exponents are found by nonlinear fitting, with the target of maximizing the $R^{2}$ values for the data sets. Both dimensionless ligament length (9) and dimensionless arithmetic mean diameter (10) are in good agreement with the depicted correlations, which are given by

$$
\begin{aligned}
\Pi_{1, \text { total }}= & \frac{l_{S e r}}{R} \cdot \frac{\rho_{g}}{\rho_{p}} \cdot \frac{\eta_{g}}{\eta_{s}} \cdot\left(\frac{c_{a x}}{\omega \cdot R}\right)^{0.030} \\
& \cdot W e_{a x, l}^{0.50} \cdot R e_{a x, l}^{-1.00} \cdot D e_{c a p}^{0.009} \cdot(1+\phi)^{-1.20}
\end{aligned}
$$

$$
\begin{aligned}
\Pi_{2, \text { total }}= & \frac{l_{\text {Ser }}}{R} \cdot \frac{\rho_{g}}{\rho_{p}} \cdot \frac{\eta_{g}}{\eta_{s}} \cdot\left(\frac{c_{a x}}{\omega \cdot R}\right)^{0.125} \\
& \cdot W e_{a x, l} 0^{0.43} \cdot R e_{a x, l}^{-0.98} \cdot D e_{c a p}^{0.004} \cdot(1+\phi)^{0.13}
\end{aligned}
$$

Both correlations are equally dependent on the ratios of densities, which result from the elimination of the

Table 1: Liquid properties for the different mixtures $S_{1}-S_{5}$ of Sterocoll ${ }^{\circledR}$ SHT and SXT used in the experimental setup

\begin{tabular}{llllllll} 
Liquid & SHT (vol.\%) & SXT (vol.\%) & $\phi\left(\mathrm{m}^{3} / \mathrm{m}^{3}\right)$ & $\rho_{I}\left(\mathrm{~kg} / \mathrm{m}^{3}\right)$ & $\sigma(\mathrm{mN} / \mathrm{m})$ & $\eta_{S}\left(\dot{\gamma}=3 \times 10^{5} \mathrm{~s}^{-1}\right)(\mathrm{Pa} \cdot \mathrm{s})$ & $\lambda_{e}(\mathrm{~s})$ \\
\hline$S_{1}$ & 2.3 & 0 & 0 & $1025.7 \pm 4.8$ & $33.4 \pm 0.3$ & $2.65 \times 10^{-2} \pm 5 \times 10^{-4}$ & $0.013 \pm 0.001$ \\
$S_{2}$ & 1.8 & 0.22 & 0 & $1013.7 \pm 6.5$ & $32.2 \pm 0.3$ & $2.57 \times 10^{-2} \pm 5 \times 10^{-4}$ & $0.038 \pm 0.002$ \\
$S_{3}$ & 1.6 & 0.40 & 0 & $1016.3 \pm 5.3$ & $33.0 \pm 0.4$ & $2.61 \times 10^{-2} \pm 1 \times 10^{-4}$ & $0.420 \pm 0.071$ \\
$S_{4}$ & 1.0 & 0.23 & 0 & $1013.7 \pm 5.4$ & $34.6 \pm 0.4$ & $2.01 \times 10^{-2} \pm 9 \times 10^{-4}$ & $0.044 \pm 0.003$ \\
$S_{5}$ & 0.2 & 0.51 & 0 & $1010.5 \pm 6.7$ & $35.2 \pm 0.2$ & $1.57 \times 10^{-2} \pm 2 \times 10^{-4}$ & $0.034 \pm 0.002$ \\
$S_{2} G_{1}$ & 1.8 & 0.22 & 1.3 & $1030.7 \pm 6.5$ & $32.7 \pm 0.3$ & $2.65 \times 10^{-2} \pm 5 \times 10^{-4}$ & $0.052 \pm 0.005$ \\
$S_{2} G_{2}$ & 1.8 & 0.22 & 3.5 & $1065.7 \pm 6.5$ & $32.2 \pm 0.3$ & $2.65 \times 10^{-2} \pm 5 \times 10^{-4}$ & $0.136 \pm 0.012$
\end{tabular}

Shear viscosities have been measured at the aforementioned shear rate by use of a piston-driven capillary rheometer at $T=25^{\circ} \mathrm{C}^{10}$ 


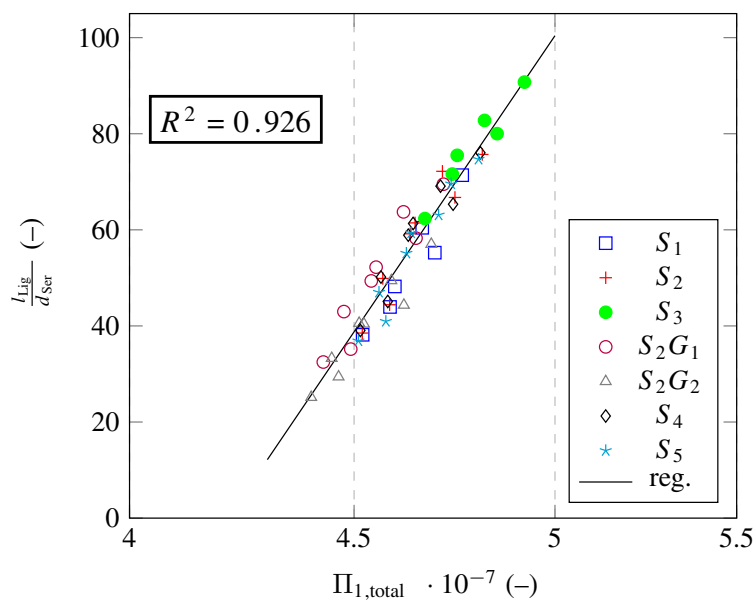

(a) Scaled ligament length over $\Pi_{1, \text { total }}$.

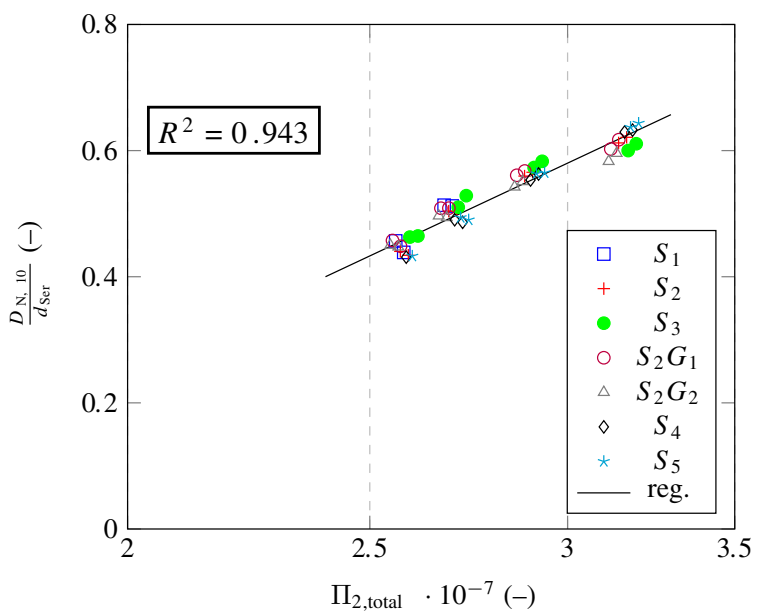

(b) Scaled arithmetic mean droplet size over $\Pi_{2, \text { total }}$.

Fig. 1: Scaled ligament length and scaled droplet size plotted against the corresponding model data from $\Pi_{1, \text { total }}$ and $\Pi_{2, \text { total }}$. Each data point represents the arithmetic average value of at least $\mathbf{1 0 . 0 0 0}$ singular detections for both ligament lengths and droplet diameters

liquid density in these terms to a linear dependency on the gas to solid density ratios and the ratios of viscosities. Increasing the solid content $\phi$ leads to shorter filaments and marginally larger droplets within the range of variation studied. The addition of glass flakes leads to occasional ligament breakup before reaching the critical breakup diameter and breakup length and, therefore, to an increase of the amount of larger droplets.

The Deborah numbers, representing the elongation flow properties, show slight influences on the ligament length and droplet size. For both correlations, the combination $\left(c_{a x} /(\omega \cdot R)\right)^{-1} \cdot W e_{a x, l}^{0.5}$ is approximately proportional to the product of the capillary breakup time $t_{c a p}$ and the strain rate $\dot{\varepsilon}=\left(R \cdot \omega^{2}\right) / c_{a x}$ as described by Kuhnhenn et al. ${ }^{6}$ The Reynolds numbers for both correlations are of equal power as the inverse viscosity ratios, thus eliminating a major influence of the shear viscosity for both droplet size and filament length and again emphasizing the importance of the gas viscosity for the breakup process. Droplet sizes and ligament length are both influenced by $c_{a x} /(\omega \cdot R)$, resulting in increasing values with increasing ratios of axial to tangential velocities.

The relative errors for both quantities can be calculated from the absolute deviations of the liquid property measurements and from detailed process parameter information by applying the variance formula to correlations (9) and (10), as all bases are independent. The relative uncertainties for the volumetric flow rate $(\Delta \dot{V} / \dot{V})$, the rotational speed $(\Delta \omega / \omega)$, the elongation relaxation time $\left(\Delta \lambda_{e} / \lambda_{e}\right)$, and the solid content $(\Delta \phi / \phi)$ are of the order $\pm 1.0 \%$. The fabrication of bell cups is a high-precision process due to the required rotational symmetry of the spinning bell cup. The systematic errors for the bell radius as well as serration dimensions can, therefore, be neglected. The overall uncertainty for the calculated ligament length is $\pm 3.95 \%$ and for the mean arithmetic diameter $\pm 1.50 \%$. The maximum deviations are $12.1 \%$ and $7.5 \%$, respectively.

\section{Conclusions}

The present study, based on dimensional analysis, including carefully determined relevant material properties and process parameters, provides a set of dimensionless groups to fully characterize the ligament and droplet formation during high-speed bell atomization in terms of the underlying physical effects, including the influences of strain rates on rheological behavior. Equation (10) can be interpreted as an extension of the solution proposed by Kuhnhenn et al., ${ }^{6}$ additionally taking into account the solid content of particles in the suspension, as well as the elongation relaxation time in form of a Deborah number. The present model is fully based on the dimensional analysis and needs to be investigated further analytically. However, our experimental results agree well with correlations (9) and (10). The present method could also be applicable to nonserrated bells, with limitations for ideal ligament buildup and breakup due to the chaotic nature of the process, as well as atomization processes under influence of electrostatic effects, effectively adding another dimensionless group.

Acknowledgments Open Access funding provided by Projekt DEAL. The authors want to thank Georg Wigger and Daniel Briesenick for the encompassing support, BASF Coatings $\mathrm{GmbH}$ for initiating this investigation, and BASF SE for allocating the material. The funding company, BASF Coatings $\mathrm{GmbH}$, did not take any influence on the design of the study, in the collection, analysis, or interpretation of data. 
Open Access This article is licensed under a Creative Commons Attribution 4.0 International License, which permits use, sharing, adaptation, distribution and reproduction in any medium or format, as long as you give appropriate credit to the original author(s) and the source, provide a link to the Creative Commons licence, and indicate if changes were made. The images or other third party material in this article are included in the article's Creative Commons licence, unless indicated otherwise in a credit line to the material. If material is not included in the article's Creative Commons licence and your intended use is not permitted by statutory regulation or exceeds the permitted use, you will need to obtain permission directly from the copyright holder. To view a copy of this licence, visit http://creativecommons.org/licenses/by/4.0/.

\section{Appendix}

See Tables 2 and 3.

Table 2: Calculated mean arithmetic diameters for the different liquids $S_{1}$ to $S_{5}$ and parameters used in the dimensional analysis

\begin{tabular}{lllll} 
Liquid & $n(\mathrm{krpm})$ & $\dot{V}_{l}(\mathrm{~mL} / \mathrm{min})$ & $D_{N, 10}(\mu \mathrm{m})$ & $I_{\text {Lig }}(\mathrm{mm})$ \\
\hline$S_{1}$ & 10 & 200 & - & 6.7 \\
$S_{1}$ & 20 & 200 & - & 5.9 \\
$S_{1}$ & 35 & 200 & 62.6 & 4.7 \\
$S_{1}$ & 50 & 200 & 55.7 & - \\
$S_{1}$ & 10 & 350 & - & 8.7 \\
$S_{1}$ & 20 & 350 & - & 7.4 \\
$S_{1}$ & 35 & 350 & 62.5 & 5.4 \\
$S_{1}$ & 50 & 350 & 53.5 & - \\
$S_{2}$ & 10 & 200 & 74.7 & 8.1 \\
$S_{2}$ & 20 & 200 & 68.3 & 7.5 \\
$S_{2}$ & 35 & 200 & 61.5 & 6.1 \\
$S_{2}$ & 50 & 200 & 53.7 & 4.7 \\
$S_{2}$ & 10 & 350 & 75.8 & 9.2 \\
$S_{2}$ & 20 & 350 & 69.0 & 8.8 \\
$S_{2}$ & 35 & 350 & 61.5 & 7.2 \\
$S_{2}$ & 50 & 350 & 53.0 & 5.4 \\
$S_{3}$ & 10 & 200 & 73.2 & 9.8 \\
$S_{3}$ & 20 & 200 & 69.9 & 9.2 \\
$S_{3}$ & 35 & 200 & 62.2 & 7.6 \\
$S_{3}$ & 50 & 200 & 56.5 & - \\
$S_{3}$ & 10 & 350 & 74.5 & 11.1 \\
$S_{3}$ & 20 & 350 & 71.1 & 10.1 \\
$S_{3}$ & 35 & 350 & 64.5 & 8.7 \\
$S_{3}$ & 50 & 350 & 56.7 & - \\
$S_{4}$ & 10 & 200 & 76.8 & 8.0 \\
$S_{4}$ & 20 & 200 & 67.5 & 7.5 \\
$S_{4}$ & 35 & 200 & 59.8 & 6.1 \\
$S_{4}$ & 50 & 200 & 52.6 & 4.8 \\
\hline & & & &
\end{tabular}

Table 2: continued

\begin{tabular}{lllll} 
Liquid & $n(\mathrm{krpm})$ & $\dot{V}_{I}(\mathrm{~mL} / \mathrm{min})$ & $D_{N, 10}(\mu \mathrm{m})$ & $I_{\text {Lig }}(\mathrm{mm})$ \\
\hline$S_{4}$ & 10 & 350 & 77.2 & 9.3 \\
$S_{4}$ & 20 & 350 & 68.7 & 8.4 \\
$S_{4}$ & 35 & 350 & 59.3 & 7.2 \\
$S_{4}$ & 50 & 350 & - & 5.5 \\
$S_{5}$ & 10 & 200 & 77.7 & 8.5 \\
$S_{5}$ & 20 & 200 & 68.7 & 7.2 \\
$S_{5}$ & 35 & 200 & 60.2 & 5.7 \\
$S_{5}$ & 50 & 200 & 52.8 & 4.5 \\
$S_{5}$ & 10 & 350 & 78.5 & 9.1 \\
$S_{5}$ & 20 & 350 & 68.9 & 7.7 \\
$S_{5}$ & 35 & 350 & 59.8 & 6.7 \\
$S_{5}$ & 50 & 350 & - & 5.0 \\
\hline
\end{tabular}

The filament lengths are obtained from high-speed imaging and evaluated by the use of the aforementioned code. Empty fields are due to insufficient count rates, filament measurability, and problems during atomization

Table 3: Calculated mean arithmetic diameters for the different liquids $S_{2} G_{1}$ and $S_{2} G_{2}$ and parameters used in the dimensional analysis

\begin{tabular}{cllll} 
Liquid & $n(\mathrm{krpm})$ & $\dot{V}_{l}(\mathrm{~mL} / \mathrm{min})$ & $D_{N, 10}(\mu \mathrm{m})$ & $I_{\text {Lig }}(\mathrm{mm})$ \\
\hline$S_{2} G_{1}$ & 10 & 200 & 73.5 & 7.1 \\
$S_{2} G_{1}$ & 20 & 200 & 68.4 & 6.4 \\
$S_{2} G_{1}$ & 35 & 200 & 62.1 & 5.2 \\
$S_{2} G_{1}$ & 50 & 200 & 55.8 & 4.0 \\
$S_{2} G_{1}$ & 10 & 350 & 75.3 & 8.5 \\
$S_{2} G_{1}$ & 20 & 350 & 69.3 & 7.8 \\
$S_{2} G_{1}$ & 35 & 350 & 62.1 & 6.0 \\
$S_{2} G_{1}$ & 50 & 350 & 54.7 & 4.3 \\
$S_{2} G_{2}$ & 10 & 200 & 71.1 & 5.4 \\
$S_{2} G_{2}$ & 20 & 200 & 66.2 & 4.9 \\
$S_{2} G_{2}$ & 35 & 200 & 60.6 & 4.1 \\
$S_{2} G_{2}$ & 50 & 200 & 54.9 & 3.1 \\
$S_{2} G_{2}$ & 10 & 350 & 72.7 & 7.0 \\
$S_{2} G_{2}$ & 20 & 350 & 67.2 & 6.0 \\
$S_{2} G_{2}$ & 35 & 350 & 60.3 & 5.0 \\
$S_{2} G_{2}$ & 50 & 350 & 54.6 & 3.6 \\
\hline
\end{tabular}

The filament lengths are obtained from high-speed imaging and evaluated by the use of the aforementioned code

\section{References}

1. Hinze, JO, Milborn, H, "Atomization of Liquids by Means of a Rotating Cup." J. Appl. Mech. Trans. ASME, 17 (2) 145-153 (1950)

2. Domnick, J, Scheibe, A, Ye, Q, "The Simulation of the Electrostatic Spray Painting Process with High-Speed Rotary Bell Atomizers. Part I: Direct Charging." Part. Part. Syst. Charact., 22 (2) 141-150 (2005)

3. Mescher, A, "Einfluss der Gasführung in Sprühtrocknern auf den Fadenzerfall an Rotationszerstäubern." PhD, TU Dortmund, 2012

4. Kamplade, J, Mack, T, Küsters, A, Walzel, P, "Break-Up of Threads from Laminar Open Channel Flow Influenced by 
Cross-wind Gas Flow." 4th Joint US-European Fluids Engineering Division Summer Meeting Collocated with the 12th International Conference on Nanochannels, Microchannels, and Minichannels, ASME, 2014

5. Kalmbach, T, Gramlich, S, Piesche, M, "Movement and Hydrodynamic Instabilities of Particle-Laden Liquid Jets in the Centrifugal Field Influenced by a Gas Flow." In: Fritsching U (ed) Process-Spray. Springer, Cham (2016). h ttps://doi.org/10.1007/978-3-319-32370-1

6. Kuhnhenn, M, Joensen, TV, Reck, M, Roisman, IV, Tropea, C, "Study of the Internal Flow in a Rotary Atomizer and Its Influence on the Properties of the Resulting Spray." Int. J. Multiph. Flow, 100 30-40 (2018)

7. Rayleigh, L, "On the Instability of Jets." Proc. Lond. Math. Soc., 1 (1) 4-13 (1878)

8. Tratnig, A, Brenn, G, Strixner, T, "Characterization of Sprays of Opaque Liquids from Pressure-Swirl Atomizers Using Phase-Doppler Anemometry." Proc. International Symposium on Applications of Laser Techniques to Fluid Mechanics, 2008

9. Oswald, W, Willenbacher, N, "Controlling the Elongational Flow Behavior of Complex Shear Thinning Fluids Without Affecting Shear Viscosity." Rheol. Acta, 58 687-698 (2019). h ttps://doi.org/10.1007/s00397-019-01170-y

10. Oswald, W, Gödeke, L, Ehrhard, P, Willenbacher, N, "Influence of the Elongational Flow Resistance and Pig- mentation of Coating Fluids on High-speed Rotary Bell Atomization." At. Sprays, 29 (10) 913-935 (2019). https://doi. org/10.1615/AtomizSpr.2020031712

11. Buckingham, E, "On Physically Similar Systems; Illustrations of the Use of Dimensional Equations." Phys. Rev., 4 (4) 345 (1914)

12. Macosko, CW, Rheology: Principles, Measurements, and Applications. Wiley-VCH, New York, 1994

13. Stelter, M, Brenn, G, Yarin, AL, Singh, RP, Durst, F, "Validation and Application of a Novel Elongational Device for Polymer Solutions." J. Rheol., 44 595-616 (2000). http s://doi.org/10.1122/1.551102

14. Weckerle, G, "Beschichtung hochwertiger Karosserieoberflächen mit Pulver-Slurry." PhD, University of Stuttgart, 2003

15. Adams, AJ, Chappie, AC, Hall, FR, "Droplet Spectra for Some Agricultural Fan Nozzles, with Respect to Drift and Biological Efficiency." In: Pesticide Formulations and Application Systems: 10th Volume. ASTM International (1990). h ttps://doi.org/10.1520/STP25379S

Publisher's Note Springer Nature remains neutral with regard to jurisdictional claims in published maps and institutional affiliations. 